\title{
The first epidemiology study of urolithiasis in New Brunswick
}

\author{
Vera Y. Chen ${ }^{1}$; Yu Chen, MD, $\mathrm{PhD}^{1,2}$ \\ ${ }^{1}$ Department of Laboratory Medicine, Dr. Everett Chalmers Regional Hospital, Horizon Health \\ Network, Fredericton, NB, Canada; ${ }^{2}$ Department of Pathology, Dalhousie University, Halifax, NS, \\ Canada
}

Acknowledgments: The authors sincerely thank James Whitman and Christiane Laforge for their excellent technical support.

Cite as: Chen VY, Chen Y. The first epidemiology study of urolithiasis in New Brunswick. Can Urol Assoc J 2020 December 29; Epub ahead of print. http://dx.doi.org/10.5489/cuaj.6888

Published online December 29, 2020

$* * *$

\section{Abstract}

Introduction: Understanding the composition of a kidney stone is crucial in leading to proper treatment and preventing reoccurring urolithiasis. This study aimed to investigate the prevalence of urolithiasis in the province of New Brunswick (NB), Canada.

Methods: A total of 3828 kidney stone analysis reports from October 1, 2016 to September 30, 2019, were reviewed from laboratory information systems. Among them, 3311 were identified as new cases. Stone compositions were analyzed by the Fourier Transform Infrared spectrometry. Incident rates were compared using Chisquared analysis of different age, sex, and regional health authority (RHA) zones.

Results: The prevalence of urolithiasis in NB was 147.8 per 100000 person-years. Males had a significantly higher $\left(\mathrm{X}^{2}=254, \mathrm{p}<0.001\right)$ incident rate of $189(95 \%$ confidence interval [CI] 182-198) than females (107 [95\% CI 102-114]) per 100000 person-years. Zone 1 had significantly higher (245 per 100000 person-years, $\mathrm{p}<0.001)$ prevalence compared to other RHA zones. Age group over 65 years had the highest incidence rate of 253 per 10000 person-years of all groups. The predominant 
kidney stone types in NB were calcium oxalate monohydrate $(60.68 \%)$ and calcium oxalate dihydrate $(11.58 \%)$. Those patients aged $0-18$ years had a high percentage of struvite $(4.32 \%)$ vs. the provincial average $(2.19 \%)(p<0.001)$.

Conclusions: The prevalence of NB's urolithiasis is slightly higher than that of Ontario. Since both zones 1.1 and 1.2 have significantly high prevalence and are situated in the Moncton area (combined zone 1), it may suggest that geographical factors play a role in the prevalence of urolithiasis in NB.

\section{Introduction}

Kidney stone disease, or urolithiasis, is common, with an overall prevalence of 1 $15 \% .{ }^{1}$ The prevalence varies significantly across geographic areas: $1-5 \%$ in Asia (except for some countries, such as Saudi Arabia, where the rate is 20\%); 5-9\% in Europe; $12 \%$ in Canada; and $13-15 \%$ in the U.S. ${ }^{2,3}$ Literature has suggested that certain factors affect the prevalence of urolithiasis. For example, males tend to get urolithiasis more often than females, with a ratio of $3: 1 .^{1}$ Age and diet may also play a role in the incidence of urolithiasis. ${ }^{4,5}$ Geographically, there is even a so-called "stone belt" in the Southeastern states of America that has a higher prevalence than other states. ${ }^{5}$ People may be predisposed to urolithiasis by a range of metabolic, infectious, genetics, and dietary conditions. In addition, environmental factors and climate characteristics may also play a role in the prevalence of this disease.

Kidney stones are not one single disease. About $70 \%$ of kidney stones are composed of calcium mostly along with oxalate, $15 \%$ contain hydroxyapatite, $10 \%$ contain uric acid; 5-10\% contain magnesium ammonium phosphate; and less than 1\% are composed of cystine. ${ }^{6,7}$ Different stones have their own treatment regimens, therefore, understanding stone composition is necessary to guide proper treatment and understanding different risk factors may help with risk stratification and recurrence prevention. ${ }^{8}$ In Canada, such epidemiology studies of urolithiasis have been conducted in Newfoundland and Labrador ${ }^{9}$ and Ontario; ${ }^{4}$ however, in New Brunswick, such data is lacking.

\section{Methods}

\section{Subjects}

A total of 3828 kidney stone analysis reports from October 1, 2016 to September 30, 2019, were reviewed from the laboratory information systems (Meditech) at the Dr. Everett Chalmers regional hospital (Fredericton, New Brunswick) and Dr. Georges-L- 
Dumont University Hospital Centre (Moncton, New Brunswick). These included urolithiasis cases from stone procedures and from those patients who spontaneously pass out in the whole province. From chart reviews, 517 cases were excluded from the study: 346 cases were repeated analyses with same stone compositions and 171 cases were not identified as urolithiasis stones (these were a combination of quartz stone samples, blood clots, gallstones, feces, or samples insufficient for analysis, etc.). There were, therefore, 3311 new urolithiasis cases over this three-year period. This study was approved by the Research Ethics Board of Horizon Health Network (File \#100577).

\section{Urolithiasis analysis}

Fourier transform infrared spectrometry (FT-IR) was the technology used to identify the different types of kidney stones as calcium oxalate monohydrate, calcium oxalate dihydrate, apatite/hydroxyapatite, struvite (magnesium ammonium phosphate hexahydrate), urate, brushite (calcium hydrogen phosphate dihydrate), cystine, dahllite (carbonate apatite), and newberyite (magnesium hydrogen phosphate trihydrate), etc. The FT-IR is semiquantitative. If a mixed stone with $70 \%$ of calcium oxalate monohydrate and $30 \%$ of apatite, the composition would be counted as a 0.7 case of calcium oxalate monohydrate and 0.3 case of apatite.

\section{Data analysis and statistics}

The disease incident rates and stone compositions were sorted by different region health authority (RHA) zones according to where the samples were submitted for analysis, i.e., Zone 1.1 (Moncton South East), Zone 2 (Saint John), Zone 3

(Fredericton), and Zone 7 (Miramichi), which are predominantly anglophone; Zone 1.2 (Beauséjour-Moncton), Zone 4 (Edmundston), Zone 5 (Campbellton), and Zone 6 (Bathurst) are mainly made up of francophones. Provincial and each RHA zone population data was acquired from Statistics Canada's 2016 Census. ${ }^{10}$ Data was also sorted by gender and age (0-18, 19-39, 40-64, and 65+ years) using an Excel spreadsheet (Microsoft, WA, U.S.). The incident rates were compared using Chisquared analysis by the SPSS version 25.0 statistical software package (SPSS Inc., Chicago, IL, U.S.). All significance tests were two-tailed using $\alpha=0.05$. 


\section{Results}

\section{Urolithiasis prevalence in New Brunswick}

The overall prevalence of urolithiasis in New Brunswick was 147.8 per 100000 person-year. Males had a significantly higher $\left(X^{2}=254, \mathrm{p}<0.001\right)$ incident rate of 189 (95\% confidence interval [CI] 182-198) per 100000 person-year compared to females, who had an incident rate of 107 (95\% CI 102-114) per 100000 person-year (Fig. 1A).

Among the four age groups, those aged 65+ had an incident rate of 253 per 100 000; those aged 40-64 had a rate of 192 per 100 000; those aged 19-39, a rate of 105 per 100 000; and those 0-18 years, eight per 100000 person-year (Fig. 1B).

Stone prevalence for the eight NB RHA zones is reported in Figs. 1C and 1D; the Moncton area rate was significantly higher than all other zones $(\mathrm{p}<0.001)$.

\section{Compositions of urolithiasis in New Brunswick (total and sexes)}

Most kidney stone types in New Brunswick were calcium oxalate monohydrate $(60.68 \%)$, followed by calcium oxalate dihydrate $(11.58 \%)$, apatite $(12.76 \%)$, urate $(9.53 \%)$, struvite $(2.19 \%)$, brushite $(1.87 \%)$, dahllite $(0.89 \%)$, cystine $(0.48 \%)$, and newberyite $(0.006 \%)$. Male New Brunswickers had a significantly lower proportion of struvite $(1.95 \%$ vs. $2.61 \%)$ compared with females, whereas higher proportion of calcium oxalate monohydrate $(63.90 \%$ vs. $55.34 \%)$ and urate $(10.50 \%$ vs. 7.92\%) (Fig. 2). The comparison of urolithiasis compositions in different RHA zones is shown in Fig. 3.

\section{Comparison of urolithiasis compositions in different age groups}

Patients aged 65+ had a high percentage of urate (15.42\%) and struvite (2.28\%), a low percentage of calcium oxalate dihydrate (8.36\%). Those aged 40-64 had similar urolithiasis proportions compared to the overall New Brunswick population. Those 19-39 years had a high percentage of apatite (22.10\%) and very little urate (1.76\%). Younger patients (ages $0-18$ had a low percentage of calcium oxalate monohydrate (39.60\%), calcium oxalate dihydrate (34.73\%), and urate $(0.54 \%)$ but the highest percentage of struvite (4.32\%) (Fig. 4).

\section{Discussion}

This study looks at the high prevalence of urolithiasis in NB compared to other Canadian provinces. One explanation could be that our study includes urolithiasis cases from stone procedures and from those patients who spontaneously pass out, 
whereas the Ontario study only included the former. Consistent with Ontario and Newfoundland and Labrador, ${ }^{4,9}$ males in NB had a significantly higher prevalence of stones than females. Different from the Ontario data, however, is that in NB, the highest prevalence of urolithiasis was seen in the older age group (those over 65 years vs. those 40-64 years in Ontario). Consistent with the literature, calcium oxalate stones (both monohydrate and dihydrate) were the predominant kidney stone types in NB.

It is noted that females had a higher incidence of struvite but lower urate compared to men (Fig. 2). This may be related to physiological sex differences, the fact that women are more prone to infections, and metabolic conditions. The elderly $(65+)$ and young children $(0-18)$ had a higher frequency of urate and struvite stones (Fig. 4). Perhaps as a result of age, these groups are more prone to infections. Young adults (19-39) had a high prevalence of apatite. While the underlying reason is not clear, age and sex have a marked influence on the type of kidney stone formed. ${ }^{11}$ This could be a focus of future research. Similarly, metabolic risk factors, such as hypercalciuria and hyperoxaluria, in different age and sex groups on the prevalence of urolithiasis is worthy of further study as well. ${ }^{12}$

People in Zone 1.2 had a significantly higher prevalence than other regions (Fig. 3). Initially, it was thought that perhaps culture and ethnicity played a role, as Zone 1.2 is a francophone region. However, other francophone regions, such as Zones 4 and 6 , had low prevalence. Both Zones 1.1 and 1.2 had significantly high rates of urolithiasis and are situated in the Moncton area (combined Zone 1), which may suggest geographical factors play a role.

One interesting finding was the $546(14.3 \%)$ repeated kidney stone analyses with no change of compositions among the total cases of 3838 . A study conducted by the Calgary Laboratory Services in 2016 found $15.9 \%$ of repeated renal calculi tests within 4.8 years $\left(87.8 \%\right.$ were repeated within three years) in Alberta. ${ }^{13}$ Furthermore, the repeated test demonstrated only rarely $(5.5 \%)$ changed kidney stone compositions. ${ }^{13}$ The consistency of our data with the previous study indicates that most repeat urolithiasis testing may not be medically necessary in the short-term, e.g. within three years, and that there might be a laboratory test use issue.

Our study is not without limitations, one of which is the missing data, e.g., patients that passed a stone and were insufficient for analysis, which might have contributed to an underestimation of disease prevalence. However, all excluded cases (171, a combination of quartz stone samples, blood clots, gallstones, feces, or samples insufficient for analysis, etc) only made up $4 \%$ of total cases (3828), therefore, we do 
not consider this potential underestimation significant. Urologists and general practitioners routinely send urinary stones to laboratory for analysis, therefore, we consider the missing data from this perspective minimal.

\section{Conclusions}

This first-ever epidemiology study of urolithiasis in NB has demonstrated a slightly higher prevalence of urolithiasis compared to that of Ontario. Since both zones in the Moncton area showed a significantly high prevalence of urolithiasis, it may suggest that geographical factors play a role. 


\section{References}

1. Pearle MS and Lotan Y: Urinary lithiasis: etiology, epidemiology, and pathogenesis. In: Campbell-Walsh Urology, $9^{\text {th }}$ ed. Edited by AJ Wein, LR Kavoussi, AC Novick et al. Philadelphia: Saunders Elsevier 2007; vol. 2, pp 1386-1387.

2. Ramello A, Vitale C, Marangella M. Epidemiology of nephrolithiasis. $J$ Nephrol. 2000;13:S45-50.

3. Michelle López, Bernd Hoppe. History, epidemiology and regional diversities of urolithiasis. Pediatr Nephrol 2010;25:49-59.

4. Ordon M, Urbach D, Mamdani M, et al. A population based study of the changing demographics of patients undergoing definitive treatment for kidney stone disease. J Urol 2015; 193:869-74.

5. Sharma AP, Filler G. Epidemiology of paediatric urolithiasis. Indian J Urol 2010; 26: 516-522.

6. Schubert G. Stone analysis. Urol Res 2006; 34:146-150.

7. Kravdal G, Helgø D, Moe MK. Infrared spectroscopy is the gold standard for kidney stone analysis. Tidsskr Nor Legeforen 2015; 135:313-14.

8. Giasson J, Chen Y. Mysterious stones. Clin Chem 2014; 60:274-275.

9. Gault MH, Chafe L. Relationship of frequency, age, sex, stone weight and composition in 15,624 stones: comparison of results for 1980 to 1983 and 1995 to 1998. J Urol 2000; 164:302-7.

10. Statistics Canada 2016 Census profile of New Brunswick. https://www12.statcan.gc.ca/census-recensement/2016/dpd/prof/index.cfm?Lang=E. Accessed on February 16, 2020.

11. Lieske JC, Rule AD, Krambeck AE, et al. Stone Composition as a Function of Age and Sex. Clin J Am Soc Nephrol 2014; 9:2141-6.

12. Richman K, O'Bell J, Pareek G. The Growing Prevalence of Kidney Stones and Opportunities for Prevention. R I Med J 2014; 97:31-4.

13. Sadrzadeh SM, Orton D, Burgess E, et al. Utilization of renal calculi analyses in Calgary [abstract]. Clin Biochem 2016; 49:1429. 


\section{Figures and Tables}

Fig. 1. Urolithiasis prevalence in New Brunswick: $(\boldsymbol{A})$ male vs. female: $X^{2}=254$, $\mathrm{p}<0.001$; (B) all age group comparisons, $\mathrm{p}<0.001$; $(\boldsymbol{C})$ prevalence of different regional health zones; and $(\boldsymbol{D})$ zone 1 compared with all other zones, $\mathrm{p}<0.001$.
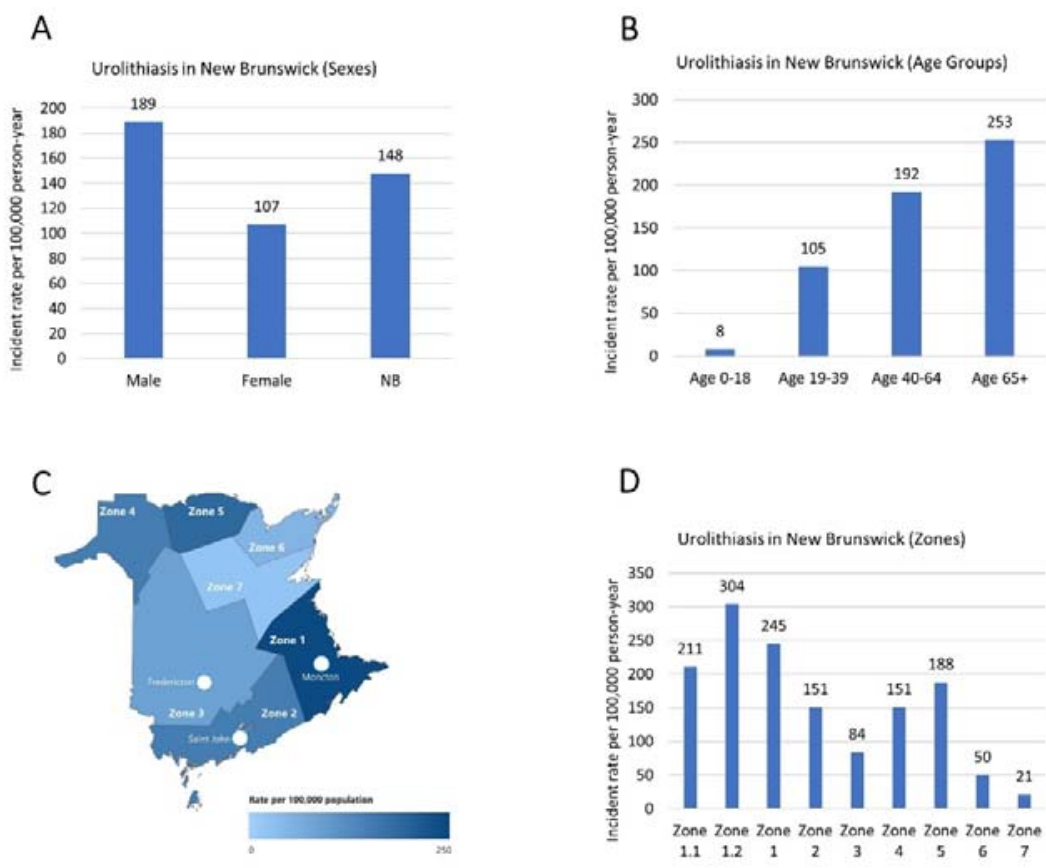

D

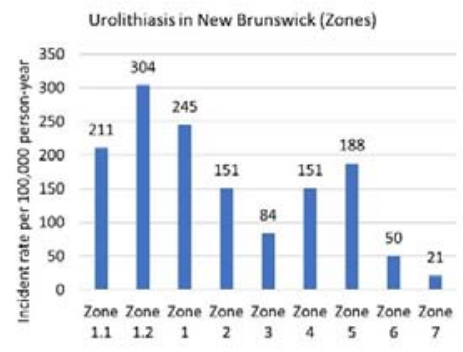

Fig. 2. Compositions of urolithiasis in New Brunswick (total and sexes).
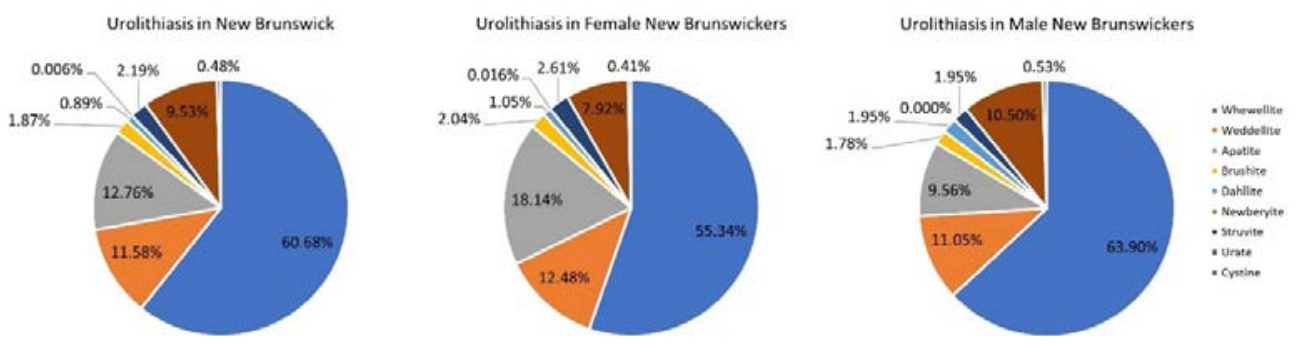
Fig. 3. Compositions of urolithiasis in New Brunswick regional health authority zones.
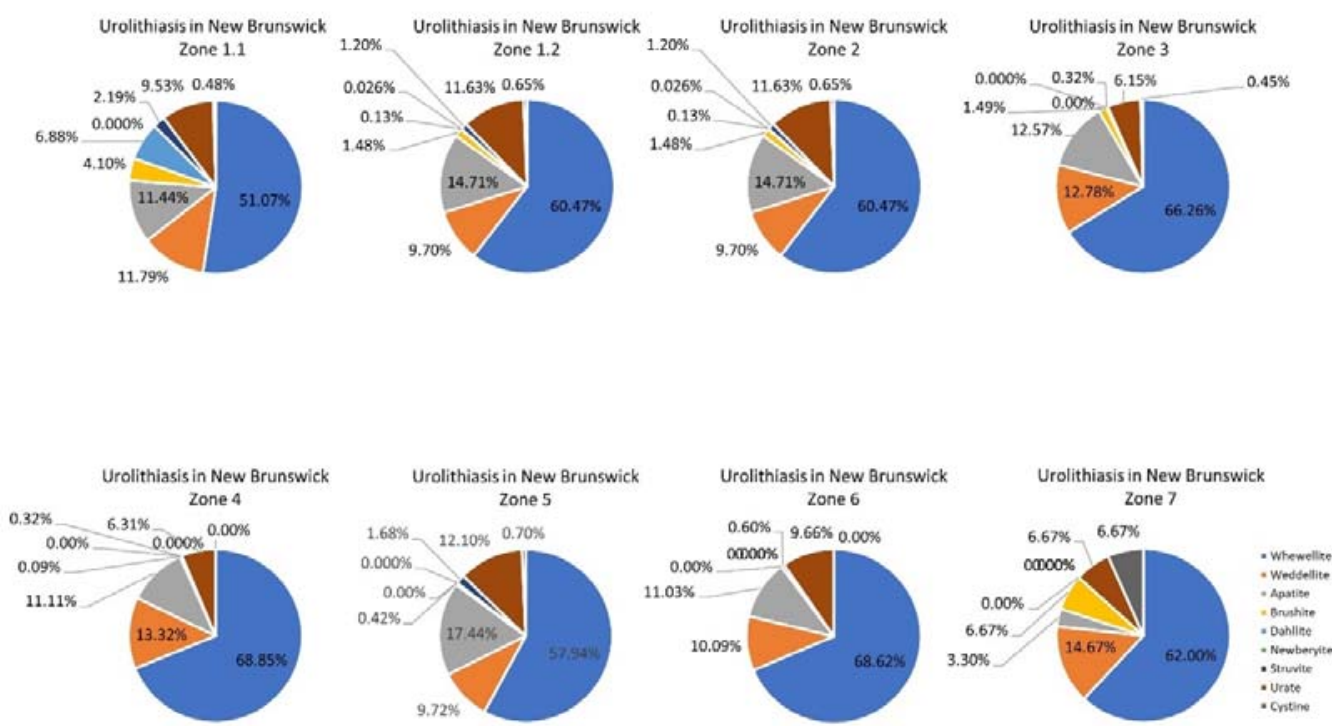

Fig. 4. Compositions of urolithiasis in New Brunswick with different age groups.
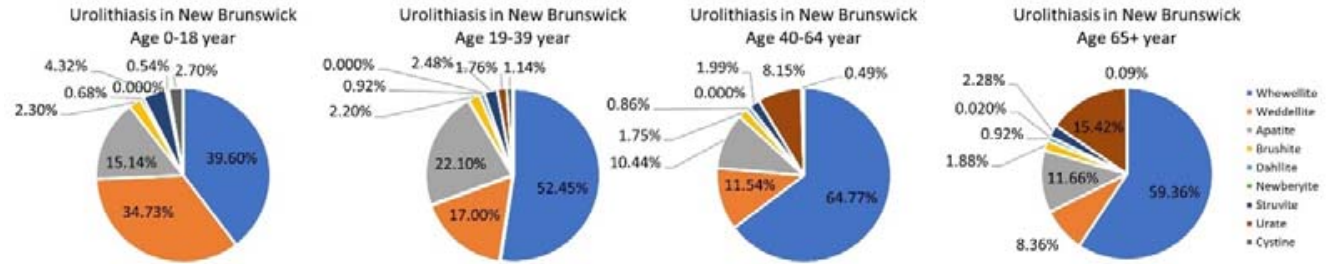